\title{
Association Between Insulin Resistance Estimated by Triglyceride Glucose Index and In-Stent Restenosis in Non-Diabetic Patients
}

\section{๑ Özge Kurmuş Ferik1, ๑ Begüm Yetiş Sayın², ๑ Kürşat Akbuğa1, ๑ Çağrı Zorlu3}

${ }^{1}$ Ufuk University Faculty of Medicine, Department of Cardiology, Ankara, Turkey

${ }^{2}$ Ankara Memorial Hospital, Clinic of Cardiology, Ankara, Turkey

${ }^{3}$ Tokat State Hospital, Clinic of Cardiology, Tokat, Turkey

\begin{abstract}
Objectives: The triglyceride glucose index (TGI) is associated with poor prognosis in cardiovascular disease. The usefulness of TGI to predict coronary in-stent restenosis (ISR) has not been determined. This study aimed to investigate the relationship between TGI and ISR in patients with stable coronary artery disease (CAD) undergoing angiography.

Materials and Methods: This retrospective study analyzed the data of 224 non-diabetic patients with coronary drugeluting stents and undergoing angiography. The patients were divided into two groups based on the angiogram results: the non-ISR group ( $\mathrm{n}=114)$ and the ISR group $(\mathrm{n}=100)$. TGI was compared between the two groups. The clinical characteristics and laboratory data were considered for univariate and multivariate analyses.

Results: No significant differences in age, sex, hypertension, and smoking history were found between the ISR and nonISR groups. TGI was higher in the ISR group than in the non-ISR group $(\mathrm{p}=0.011)$. According to the multiple logistic regression analysis, Gensini score and SYNTAX score, TGI and white blood cell count were independent predictors of ISR.

Conclusion: Patients with ISR were found to have higher TGI than those without ISR, suggesting that TGI might be a valuable predictor of ISR in patients with stable CAD.
\end{abstract}

Keywords: Triglyceride glucose index, insulin resistance, in-stent restenosis

Address for Correspondence: Özge Kurmuş Ferik, Ufuk University Faculty of Medicine, Department of Cardiology, Ankara, Turkey e-mail: ozge_kurmus@yahoo.com ORCID: orcid.org/0000-0003-2067-7492

Received: 29.11.2021 Accepted: 06.01.2022

Cite this article as: Kurmuş Ferik Ö, Yetiş Sayın B, Akbuğa K, Zorlu Ç. Association Between Insulin Resistance Estimated by Triglyceride Glucose Index and In-Stent Restenosis in Non-Diabetic Patients. EJCM 2022;10(1):12-17.

DOI: 10.32596/ejcm.galenos.2022.2021-11-060 


\section{Introduction}

Insulin resistance has been established as a mediator of type 2 diabetes mellitus (T2DM), metabolic syndrome, and atherosclerotic cardiovascular disease (CVD) $)^{(1,2)}$. Chronically increased plasma glucose and triglyceride levels are associated with insulin resistance ${ }^{(2)}$. Triglyceride glucose index (TGI), a product of plasma glucose and triglyceride levels, has been demonstrated to be a surrogate of insulin resistance in previous studies ${ }^{(3,4)}$.

Despite the evidence that drug-eluting coronary stents reduce restenosis compared with bare-metal stents, stent restenosis is still an ongoing clinical problem ${ }^{(5)}$. Factors related to in-stent restenosis (ISR) are diverse. T2DM and hyperinsulinemia are known to be associated with increased restenosis rate after stent implantation ${ }^{(6,7)}$.

Previous studies indicated that TGI was associated with the risk of incident hypertension and incident CVD and adverse outcomes in patients with acute coronary syndromes ${ }^{(8-11)}$. No previous study exclusively investigated the usefulness of TGI in predicting ISR. Therefore, this study examined the relationship between TGI and ISR in patients undergoing coronary angiography for stable coronary artery disease (CAD).

\section{Materials and Methods}

This retrospective study assessed the data of 1,310 patients who had undergone coronary angiography between January 2019 and June 2020 in our angiography laboratory because of suspicion of stable CAD. Patients with incomplete data, diabetes mellitus (DM), prior coronary artery bypass grafting, bare-metal stent, prior intervention of ISR, systemic inflammatory disease, renal or hepatic failure, severe heart failure, hypo/ hyperthyroidism, extreme body mass index (BMI), suspected familial hypertriglyceridemia or receiving fenofibrate were not included in the study. Finally, the data of 224 patients were evaluated in the analysis. The patients were divided into two groups according to their angiography results: the non-ISR group $(n=114)$ and the ISR group $(\mathrm{n}=100)$.
All patients in the present study had previously implanted coronary drug-eluting stents in our institution. Coronary angiography was performed using the standard Judkins technique. Angiographic restenosis was defined as stenosis $\geq 50 \%$ of the lumen diameter of the previously implanted stent (ISR and/or $5 \mathrm{~mm}$ proximal and distal to the stent edge). The angiographic results and the severity of ISR were recorded from patients' files. If the patient had more than one stent, the stent with the most severe restenosis was recorded. Baseline diagnostic angiograms of the patients were assessed independently by two experienced cardiologists to evaluate the extent and severity of CAD by calculating the Gensini and SYNTAX scores. The Gensini score was calculated by assigning a severity score to each coronary narrowing on the basis of the degree of luminal stenosis and its location. Decreases in luminal diameter of $25 \%, 50 \%, 75 \%, 90 \%, 99 \%$, and total occlusion were given scores of 1, 2, 4, 8, 16 and 32, respectively. The score was then multiplied by a factor symbolizing the functional significance of the myocardial area supplied by that segment ${ }^{(12)}$. The SYNTAX score for each patient was calculated by scoring all coronary lesions producing $\geq 50 \%$ diameter stenosis in vessels $\geq 1.5 \mathrm{~mm}^{(13)}$. Stent restenosis was excluded while calculating Gensini and SYNTAX scores. The assessed clinical parameters were age, gender, weight, height, coronary risk factors, and statin use. Hypertension was defined as systolic blood pressure $\geq 140 \mathrm{mmHg}$ and/or diastolic blood pressure $\geq 90 \mathrm{mmHg}$ or current medication with antihypertensive drugs. The BMI was calculated as body weight in kilograms divided by the squared value of body height in meters $\left(\mathrm{kg} / \mathrm{m}^{2}\right)$.

Plasma lipid and plasma glucose level measurements were performed on fasting venous blood samples taken before the angiography. Plasma levels of total cholesterol, low-density lipoprotein cholesterol (LDL-C), high-density lipoprotein cholesterol (HDL-C), triglyceride and glucose were measured using a clinical biochemistry analyzer (Abbott Architect c 8000). The TGI was calculated as the "In [fasting triglyceride (mg/dL) x fasting plasma glucose $(\mathrm{mg} / \mathrm{dL}) / 2]^{\prime(14)}$. 
The study was approved by the Mersin University Clinical Research Ethics Committee (approval number: 2021/33). Patient data were analyzed retrospectively.

\section{Statistical Analysis}

Continuous variables were expressed as mean \pm standard deviation; categorical variables were defined as percentages. The distribution of continuous variables was considered normal or not based on the KolmogorovSmirnov test. The Student's t-test was used to compare continuous variables. Differences in the distribution of categorical variables were assessed by chi-square test. Univariate and multiple logistic regression analyses were used to identify the independent predictors of ISR. Variables with p-value equal to or less than 0.25 on univariate analysis were selected for multiple analysis. The Pearson's correlation analysis was employed to examine the correlation between continuous variables. A p-value of $<0.05$ was considered statistically significant. Data analyses were performed using SPSS for Windows, version 27.0 (SPSS Inc., Chicago, IL, United States).

Table 1. Demographic and laboratory data of the patients

Variable
Age (year)
Male (\%)
Hypertension (\%)
Smoking (\%)
Receiving statin (\%)
BMI (kg/m ${ }^{2}$ )
Gensini score
SYNTAX score
TGI
White blood cell count (x10 $/ \mathrm{L}$ )
Hemoglobin (g/dL)
Total cholesterol (mg/dL)
LDL-C (mg/dL)
Uric acid (mg/dL)
Creatinine (mg/dL)

ISR: In-stent restenosis, BMI: Body mass index, TGI: Triglyceride glucose index, LDL-C: Low density lipoprotein cholesterol, HDL-C: High density lipoprotein cholesterol

Significant $p$-values are shown in bold.

\section{Results}

The mean age of the study population was $64.0 \pm 10.2$ years, and $68.8 \%$ of the 224 patients were male. Moreover, $59 \%$ of the patients had hypertension, and $27 \%$ had any smoking history. The mean Gensini score was $42.8 \pm 6.5$ and the mean SYNTAX score was $13.5 \pm 6.5$. The Gensini score was highly correlated with the SYNTAX score $(\mathrm{r}=0.904, \mathrm{p}<0.001)$. The TGI weakly correlated with the Gensini and SYNTAX scores $(\mathrm{r}=0.243, \mathrm{p}=0.041, \mathrm{r}=0.247$, $\mathrm{p}=0.036$, respectively). The demographic characteristics of both the non-ISR group and ISR group are shown in Table 1. No significant differences in age, sex, hypertension, any smoking history and statin use were found between the two groups. Patients' medicines were recorded after admission to hospital and all patients found to have full compliance to antiaggregant therapy. The Gensini and SYNTAX scores were higher in the ISR group than the non-ISR group. TGI, total cholesterol, LDL-C, uric acid levels and white blood cell count were higher in the ISR group than in the non-ISR group. BMI, creatinine and hemoglobin did not differ between the groups.

\begin{tabular}{|l|l|l|}
$\begin{array}{l}\text { ISR group } \\
(\mathbf{n}=100)\end{array}$ & $\begin{array}{l}\text { Non-ISR group } \\
(\mathbf{n}=114)\end{array}$ & p-value \\
\hline $65.9 \pm 9.9$ & $63.6 \pm 10.3$ & 0.290 \\
\hline 69.0 & 74.5 & 0.366 \\
\hline 60.0 & 63.1 & 0.635 \\
\hline 27.0 & 28.9 & 0.752 \\
\hline 65.0 & 68.4 & 0.596 \\
\hline $26.8 \pm 3.2$ & $26.6 \pm 3.4$ & 0.360 \\
\hline $48.5 \pm 13.7$ & $41.6 \pm 12.8$ & $\mathbf{0 . 0 2 7}$ \\
\hline $16.2 \pm 5.5$ & $11.9 \pm 5.8$ & $\mathbf{0 . 0 3 4}$ \\
\hline $5.1 \pm 0.33$ & $4.7 \pm 0.32$ & $\mathbf{0 . 0 1 1}$ \\
\hline $8.12 \pm 1.5$ & $7.13 \pm 2.5$ & $\mathbf{0 . 0 4 0}$ \\
\hline $13.6 \pm 1.8$ & $13.8 \pm 1.9$ & 0.551 \\
\hline $185.5 \pm 21.5$ & $179.6 \pm 21.6$ & $\mathbf{0 . 0 4 5}$ \\
\hline $108.4 \pm 11.4$ & $100.3 \pm 10.4$ & $\mathbf{0 . 0 4 1}$ \\
\hline $6.19 \pm 1.5$ & $5.28 \pm 1.4$ & $\mathbf{0 . 0 1 2}$ \\
\hline $1.0 \pm 0.2$ & $1.1 \pm 0.2$ & 0.715 \\
\hline
\end{tabular}


In univariate analysis, TGI $(\mathrm{p}=0.010)$, total cholesterol $(\mathrm{p}=0.029)$, uric acid levels $(\mathrm{p}=0.044)$, white blood cell count ( $\mathrm{p}=0.018)$, SYNTAX ( $\mathrm{p}=0.001)$ and Gensini scores $(p=0.001)$ were associated with ISR. Multiple logistic regression analysis revealed that TGI $(\mathrm{p}=0.015)$, white blood cell count $(p=0.027)$, Gensini score $(p=0.001)$ and SYNTAX score $(\mathrm{p}=0.001)$ were independent predictors of ISR (Table 2). Each time TGI increased a unit, patients were 1.3 times more likely to have ISR [Odds ratio $(\mathrm{OR})=1.328,95 \%$ confidence interval $(\mathrm{CI})=1.103-1.654$; $\mathrm{p}=0.015)]$. Also, each time Gensini score increased a unit, patients were 1.2 times more likely to have ISR $(\mathrm{OR}=1.231$, 95\% CI=1.130-1.675; $\mathrm{p}=0.001)$. When SYNTAX score increased a unit, patients were 1.3 times more likely to have ISR $(\mathrm{OR}=1.301,95 \% \mathrm{CI}=1.140-1.790 ; \mathrm{p}=0.001)$.

\section{Discussion}

This study found that TGI was higher in the ISR group than in the non-ISR group. After adjusting for the confounding factors, TGI was significantly associated with ISR. Insulin resistance causes an increase in blood triglyceride and glucose levels ${ }^{(2)}$. TGI has been used as a predictor of insulin resistance in recent studies ${ }^{(3,4)}$. Also, TGI has been demonstrated to be a valuable predictor of T2DM ${ }^{(15)}$.

Recent studies have used TGI as a biomarker in patients with CVD or with metabolic risk factors. TGI has been found to be associated with subclinical atherosclerosis, arterial stiffness and incident $\mathrm{CVD}^{(9,16)}$. It has also been found to be a predictor of future cardiovascular events in patients with stable $\mathrm{CAD}^{(17)}$. In this study, patients with ISR had higher TGI compared with those without ISR.
Also, TGI correlated with the severity of atherosclerotic lesions, was evaluated as Gensini and SYNTAX scores, in the present study population.

Different clinical, angiographic and operative factors may have a role in ISR. The restenosis rate is approximately $<10 \%$ in drug-eluting stent $\mathrm{era}^{(5)}$. DM is a well-known risk factor for restenosis. Neointimal tissue proliferation is one of the mechanisms of ISR in diabetic patients ${ }^{(18)}$. An insulin-sensitizing agent, troglitazone, was shown to reduce neointimal tissue proliferation ${ }^{(19)}$. Hyperinsulinemia during the oral glucose test was found to be associated with neointimal tissue proliferation in non-diabetic patients ${ }^{(20)}$. Increased secretion of insulin during the oral glucose test was also found to be associated with angiographic restenosis in non-diabetic patients ${ }^{(9)}$. Although cumulative evidence supports that insulin resistance is associated with increased CVD morbidity and mortality regardless of DM, insulin resistance has not received much attention in nondiabetic patients with CAD. TGI has been demonstrated to be significantly associated with insulin resistance even better than homeostasis model assessment of insulin resistance (HOMA-IR) in non-diabetic patients ${ }^{(3,4)}$. HOMA-IR is a relatively extensive method and it is not easily available. TGI is a practical tool and easily available. Insulin resistance by TGI was evaluated in the present study and it was found to be an independent predictor of ISR. TGI might identify patients with higher risk, thereby bringing attention to improving of insulin resistance besides dyslipidemia in CAD patients without DM.

White blood cell count, SYNTAX and Gensini scores were also independent predictors of ISR in the present study. Our results are consistent with previous studies.

Table 2. Multiple binomial logistic regression analysis: predictors of in-stent restenosis

\begin{tabular}{|c|c|c|c|c|}
\hline Predictors & $\begin{array}{l}\text { Regression } \\
\text { coefficients }\end{array}$ & Odds ratio & $\begin{array}{l}95 \% \text { confidence } \\
\text { interval }\end{array}$ & $p$-value \\
\hline Gensini score & 0.208 & 1.231 & $1.130-1.675$ & 0.001 \\
\hline SYNTAX score & 0.263 & 1.301 & $1.140-1.790$ & 0.001 \\
\hline TGI & 0.284 & 1.328 & $1.103-1.654$ & 0.015 \\
\hline White blood cell count & 0.145 & 1.156 & $1.041-1.280$ & 0.027 \\
\hline
\end{tabular}


Higher white blood cell count is an indicator of inflammation and high levels of inflammatory factors have been found to be related to a greater risk of restenosis ${ }^{(21)}$. Higher inflammatory markers such as white blood cell count may indicate greater atherosclerotic disease activity and susceptibility to endothelial dysfunction and neointimal tissue proliferation ${ }^{(21)}$. Serum uric acid levels had been shown as a predictor for cardiovascular diseases with an increased inflammatory response. Also, some studies showed that preprocedural uric acid levels increased the bare-metal stent ISR in patients with stable and unstable angina pectoris ${ }^{(22)}$. In our study, univariate analysis showed that uric acid levels had also an impact in higher degrees of ISR. Higher Gensini and SYNTAX scores are also indicators of extensive atherosclerotic disease and ongoing, accelerated atherosclerotic process and neo atherosclerosis is one of the potential mechanisms of the restenosis ${ }^{(23)}$.

\section{Study Limitations}

This study has several limitations. The effects of all potential confounding factors on ISR could not be controlled because of the retrospective design of the study. Patients with DM and receiving fenofibrate were not included in the present study, but patients with undiagnosed DM and dyslipidemias may affect plasma glucose and triglyceride levels. Another study which includes easily available inflammatory and oxidative indices including red cell distribution width, platelet to lymphocyte ratio, neutrophil to lymphocyte ratio, monocyte to high-density lipoprotein cholesterol ratio, serum total bilirubin levels that may cause ISR could be planned and compared with TGI. Finally, this was an observational study and hence did not provide a cause-effect relationship.

\section{Conclusion}

In conclusion, the results of this study suggested that TGI was an independent predictor of ISR in stable CAD patients without DM. However, further studies are required to validate these results and determine whether the combination of high triglyceride and high glucose levels may represent an underlying mechanism for ISR.

\section{Ethics}

Ethics Committee Approval: This study was approved by Mersin University Clinical Research Ethics Committee (approval number: 2021/33).

Informed Consent: Patient data were collected retrospectively.

Peer-review: Externally peer-reviewed.

\section{Authorship Contributions}

Surgical and Medical Practices: Ö.K.F., B.Y.S., K.A., Ç.Z., Concept: Ö.K.F., B.Y.S., K.A., Ç.Z., Design: Ö.K.F., B.Y.S., K.A., Ç.Z., Data Collection and/or Processing: Ö.K.F., B.Y.S., K.A., Ç.Z., Analysis and/or Interpretation: Ö.K.F., B.Y.S., K.A., Ç.Z., Literature Search: Ö.K.F., B.Y.S., K.A., Ç.Z., Writing: Ö.K.F., B.Y.S., K.A., Ç.Z.

Conflict of Interest: The authors report conflicts of interest regarding the content herein.

Financial Disclosure: This research received no specific grant from any funding agency.

\section{References}

1. Rask-Madsen C, Kahn CR. Tissue-specific insulin signaling, metabolic syndrome, and cardiovascular disease. Arterioscler Thromb Vasc Biol 2012;32:2052-9

2. Ormazabal V, Nair S, Elfeky O, Aguayo C, Salomon C, Zuñiga FA. Association between insulin resistance and the development of cardiovascular disease. Cardiovasc Diabetol 2018;17:122.

3. Guerrero-Romero F, Simental-Mendía LE, González-Ortiz M, et al. The product of triglycerides and glucose, a simple measure of insulin sensitivity. Comparison with the euglycemic-hyperinsulinemic clamp. J Clin Endocrinol Metab 2010;95:3347-51

4. Vasques AC, Novaes FS, de Oliveira Mda S, et al. TyG index performs better than HOMA in a Brazilian population: a hyperglycemic clamp validated study. Diabetes Res Clin Pract 2011;93:e98-100.

5. Buccheri D, Piraino D, Andolina G, Cortese B. Understanding and managing in-stent restenosis: a review of clinical data, from pathogenesis to treatment. J Thorac Dis 2016;8:E1150-62.

6. Paramasivam G, Devasia T, Jayaram A, et al. In-stent restenosis of drugeluting stents in patients with diabetes mellitus: Clinical presentation, angiographic features, and outcomes. Anatol J Cardiol 2020;23:28-34.

7. Babalik E, Gürmen T, Orhan L, et al. Increased secretion of insulin during oral glucose tolerance test can be a predictor of stent restenosis in nondiabetic patients. Catheter Cardiovasc Interv 2003;58:306-12. 
8. Xie Y, Guo R, Li Z, et al. Temporal relationship between body mass index and triglyceride-glucose index and its impact on the incident of hypertension. Nutr Metab Cardiovasc Dis 2019;29:1220-9.

9. Li S, Guo B, Chen H, et al. The role of the triglyceride (triacylglycerol) glucose index in the development of cardiovascular events: a retrospective cohort analysis. Sci Rep 2019;9:7320.

10. Ma X, Dong L, Shao Q, et al. Triglyceride glucose index for predicting cardiovascular outcomes after percutaneous coronary intervention in patients with type 2 diabetes mellitus and acute coronary syndrome. Cardiovasc Diabetol 2020;19:31.

11. Luo E, Wang D, Yan G, et al. High triglyceride-glucose index is associated with poor prognosis in patients with acute ST-elevation myocardial infarction after percutaneous coronary intervention. Cardiovasc Diabetol 2019;18:150

12. Gensini GG. A more meaningful scoring system for determining the severity of coronary heart disease. Am J Cardiol 1983;51:606.

13. Serruys PW, Morice MC, Kappetein AP, et al. Percutaneous coronary intervention versus coronary-artery bypass grafting for severe coronary artery disease. N Engl J Med 2009;360:961-72.

14. Guerrero-Romero F, Simental-Mendía LE, González-Ortiz M, et al. The product of triglycerides and glucose, a simple measure of insulin sensitivity. Comparison with the euglycemic-hyperinsulinemic clamp. J Clin Endocrinol Metab 2010;95:3347-51.

15. Chamroonkiadtikun P, Ananchaisarp T, Wanichanon W. The triglycerideglucose index, a predictor of type 2 diabetes development: A retrospective cohort study. Prim Care Diabetes 2020;14:161-7.

16. Lambrinoudaki I, Kazani MV, Armeni E, et al. The TyG Index as a Marker of Subclinical Atherosclerosis and Arterial Stiffness in Lean and Overweight Postmenopausal Women. Heart Lung Circ 2018;27:716-24.
17. Jin JL, Cao YX, Wu LG, et al. Triglyceride glucose index for predicting cardiovascular outcomes in patients with coronary artery disease. J Thorac Dis 2018;10:6137-46.

18. Kornowski R, Mintz GS, Kent KM, et al. Increased restenosis in diabetes mellitus after coronary interventions is due to exaggerated intimal hyperplasia. A serial intravascular ultrasound study. Circulation 1997;95:1366-9.

19. Takagi T, Akasaka T, Yamamuro A, et al. Troglitazone reduces neointimal tissue proliferation after coronary stent implantation in patients with noninsulin dependent diabetes mellitus: a serial intravascular ultrasound study. J Am Coll Cardiol 2000;36:1529-35.

20. Takagi T, Yoshida K, Akasaka T, et al. Hyperinsulinemia during oral glucose tolerance test is associated with increased neointimal tissue proliferation after coronary stent implantation in nondiabetic patients: a serial intravascular ultrasound study. J Am Coll Cardiol 2000;36:731-8.

21. Qian H, Luo Z, Xiao C, et al. Red cell distribution width in coronary heart disease: prediction of restenosis and its relationship with inflammatory markers and lipids. Postgrad Med J 2018;94:489-94.

22. Turak O, Canpolat U, Özcan F, et al. Usefulness of preprocedural serum uric acid level to predict restenosis of bare metal stents. Am J Cardiol 2014;113:197-202

23. Wang JL, Qin Z, Wang ZJ, et al. New predictors of in-stent restenosis in patients with diabetes mellitus undergoing percutaneous coronary intervention with drug-eluting stent. J Geriatr Cardiol 2018;15:137-45. 